\title{
Discrimination Among Wave-Generated Sounds by a Swash-Riding Clam
}

\author{
OLAF ELLERS* \\ Department of Zoology, Duke University, Durham, North Carolina 27706
}

\begin{abstract}
Clams, Donax variabilis, responded to sound stimuli presented to them in a laboratory aquarium by jumping out of the sand, lying on the sand for several seconds, and digging in again. On a beach, clams jump out of the sand and ride waves, migrating shoreward with the rising tide and seaward with the falling tide. Parallels between clam behavior on a beach and that elicited in the laboratory suggest that clams cue on wave sounds to jump out of the sand. Three aspects of the response to sound were parallel. (i) Clams were most responsive to low-frequency sounds similar to those produced on a beach by waves rolling onto shore. (ii) Clams were also more responsive to louder sounds; on a beach, clams jump preferentially for the largest (loudest) $20 \%$ of waves. (iii) Responsiveness in the laboratory had an endogenous tidal rhythm, with highest activity occurring at high tide and no activity occurring at low tide; this rhythm corresponds to the activity of clams on the beach from which they were collected. By using sounds to identify large waves, clams can ride selected waves and continuously maintain position at the sea's edge as the tide floods and ebbs.
\end{abstract}

\section{Introduction}

Large populations of the coquina clam, Donax variabilis, migrate on sandy beaches, shoreward with the rising tide and seaward with the falling tide (as do many other clams in this genus). An individual D. variabilis, which can be up to $3 \mathrm{~cm}$ long, normally resides with the posterior edge of its shell about 2 to $7 \mathrm{~mm}$ under the surface of the sand. To migrate, several times each tidal cycle, it jumps out of the sand (pushing its shell upward by thrusting two to five times downward with its foot) and rides flow from

Received 9 September 1994; accepted 27 July 1995

* Current address: Section of Evolution and Ecology, Division of Biological Sciences, University of California, Davis, CA 95616. waves. This method of locomotion has been named "swash-riding" (Ellers, 1987, 1988).

Individual $D$. variabilis control where waves move them, in part, by choosing to ride specific waves (Ellers, 1995a). On a rising tide, clams jump out of the sand preferentially for the biggest waves; i.e., the ones that drag them the largest distance in the shoreward direction. Astonishingly, the clams jump out of the sand before the arrival of such waves, thus effectively predicting which waves will carry them the furthest. The cues that enable this behavior have not been previously investigated.

One explanation for this discriminatory and anticipatory feat is that clams might detect sounds from waves and use differences in the character of these sounds to select waves. Sound is a reasonable candidate as a cue that enables clams to discriminate among waves because a physical mechanism linking louder sounds to larger waves is plausible. On dissipative beaches where the clams live, a breaking wave becomes a bore (a traveling cliff-like structure of tumbling water) and the bore becomes swash, which the clams ride. The higher the bore, the larger the excursion of the swash (Bradshaw, 1982). Since a higher bore has more potential energy - it has farther to fall-it tends to create more intense vibrations as water falls off its leading edge, thus emitting a louder sound. Sound is also a reasonable cue that enables clams to anticipate waves since sound travels faster than, and in front of, the bore, thus announcing its arrival. Species of Donax respond to sound or vibration by either jumping out of the sand or burrowing more deeply (Mori, 1938, 1950; Loesch, 1957; Turner and Belding, 1957; Tiffany, 1971; Trueman, 1971), but no quantitative data describing either wave sounds or sounds that elicited responses from clams are available.

Reports of animals using flow-induced sounds as behavioral cues are very rare. Flow-induced low-frequency sounds and infrasounds emanate from weather patterns, 
topographic features, and ocean waves; such sounds can travel thousands of kilometers. Birds are able to detect infrasounds and may be able to orient relative to such features because birds can detect Doppler shifts associated with flying away from or towards infrasound sources (Kreithen and Quine, 1979). Detection of low-frequency sound and infrasound has also been shown in fish (KarIsen, 1992a, b) and cephalopods (Packard et al., 1990). The function of infrasound detection in these cases is unclear, but may be related to orientation, detection of surface waves, short-term inertial guidance, or detection of low-frequency flows (Bleckmann et al., 1991) from swimming motions of other fish. Avoidance of continuously vibrating sound sources in darkness has been demonstrated in herring (Blaxter and Batty, 1985).

Sound is mechanical energy (created by a vibration) that propagates through a medium as a result of kinetic and potential energy being alternately stored and released elastically by the medium. Sound propagates either through the air or through the wet beach sand at the speeds of sound in those media. As the sound vibrations travel past a point, they are detectable as temporal changes in velocity, displacement, or pressure; the latter can be measured using a hydrophone.

To determine whether listening for and distinguishing among waves is possible under the sand, I recorded wave sounds by using a hydrophone planted under the sand amidst a population of burrowed $D$. variabilis. Then, in a laboratory, I tested whether clams would respond by jumping out of the sand when wave-like sounds were presented in the absence of waves. I also used recorded wave sounds and several artificial sounds of varying loudness and frequency to test the frequency and loudness specificity of clams' responses.

The above experiments must be considered in the light of suggestions of a tidal rhythm of responsiveness to sound. Behavior of $D$. variabilis on a beach changes with time of tide (Ellers, 1995a), and jumping responses to vibration were observed during rising tides only (Turner and Belding, 1957) or during all tidal phases (Tiffany, 1971). An endogenous rhythm was suggested for $D$. semigranosus (Mori, 1938, 1950). Tidal rhythms are often found in coastal marine invertebrates, particularly in crustaceans, molluscs, and polychaetes (for a review, see Naylor, 1985). An endogenous tidal rhythm of shell gaping has been documented in a venerid clam (Williams et al., 1993). Therefore, I also tested for an endogenous tidal rhythm of responsiveness to sound.

\section{Materials and Methods}

\section{Specimens}

Immediately preceding each laboratory experiment, fresh Donax variabilis were collected from a beach (sub- sequently "the" beach) near Pine Knoll Shores on the seaward side of Bogue Bank, a barrier island off North Carolina. The clams were transported with sand and seawater in a thermally insulated container to the Duke University Marine Laboratory, a half-hour drive from the beach. (An insulated container is crucial; if clams heat up even slightly in transit, they subsequently show no behavioral responses.) Clams were placed in an aquarium, where they dug into sand.

\section{General acoustic tests and analyses}

Acoustic tests in the laboratory were done in a glass aquarium ( $50 \mathrm{~cm}$ long by $26 \mathrm{~cm}$ wide by $30 \mathrm{~cm}$ high) placed on a plywood table. The bottom $8 \mathrm{~cm}$ of the aquarium was covered with sand from the beach, and seawater was added to a depth of $28 \mathrm{~cm}$.

Sounds were produced in several ways. Knocking with knuckles on the plywood table produced a low-frequency sound. In addition, sounds were produced using an underwater loudspeaker ( $20 \mathrm{~cm}$ diameter) made of polystyrene foam. The loudspeaker, located $10 \mathrm{~cm}$ from one end of the aquarium and partially buried in the sand, was driven by a tape recorder. Sounds played were either recorded from waves on the beach or synthesized by a sine wave generator circuit.

To determine the sound pattern reaching the clams, sounds were recorded by hydrophones buried in the sand at various locations in the aquarium. The signal from the hydrophone was amplified and digitized (8-bit resolution) at a sampling rate of $8192 \mathrm{~Hz}$. This sampling rate allows detection of sounds below $4096 \mathrm{~Hz}$. Higher sampling rates were unnecessary because very little sound was detected at higher frequencies when higher sampling rates were tried.

To analyze the data, several standard methods were used. Sound pressure was plotted as a function of time. The pressure functions were transformed into frequency spectra in which sound amplitude is plotted as a function of frequency. This transformation was performed using a Fast Fourier Transform (FFT) computer algorithm (Burrus and Parks, 1985, p. 107). The FFT algorithm produces a complex number for each frequency; the amplitude at each frequency interval is twice the absolute value of that complex number divided by the square root of the number of samples. Frequency resolution is the reciprocal of the time interval sampled (a 0.5 -s sample of sound resolves into $2-\mathrm{Hz}$ intervals).

I represented loudness of sound as either the root-meansquare (RMS) of the pressure trace over an interval of interest or as the logarithm of that value. Loudness is a subjective concept; because humans judge the relative loudness of two sounds as the ratio of their intensities, loudness is often represented using a logarithmic (decibel) 
scale (Kinsler et al., 1982). One conventional measure of sound levels is the intensity level $(I L)$ :

$$
I L=10 \log _{10}\left(\frac{I}{I_{\mathrm{ref}}}\right)
$$

where $I$ is the measured intensity and $I_{\text {ref }}$ is a reference intensity. Intensity is the rate at which sound energy flows through a unit area. But for both plane and spherical waves,

$$
I=\frac{P_{e}^{2}}{\rho c}
$$

where $P_{\mathrm{e}}=$ RMS sound pressure, $\rho=$ density, and $c=$ the speed of sound in the medium in which the sound is traveling. Although an approximate speed of sound in wet sand is obtainable from the literature, the exact speed of sound in the wet sand in the present experiment is unknown. Therefore, I present $P_{\mathrm{e}}$ rather than the derived quantity, $I$.

Furthermore, it is not clear, a priori, that clams should respond logarithmically to sound. Therefore, I present all data numerically as either pressure or $P_{e}$, but relative loudness is assumed to be represented by the ratios of the $P_{e}$ values (reflected in a logarithmically transformed $P_{e}$ axis in graphs that include several sounds). I also give the following conversions to a common measure called the sound pressure level, SPL:

$$
\mathrm{SPL}=20 \log _{10}\left(\frac{P_{e}}{P_{\text {ref }}}\right)
$$

where $P_{\text {ref }}=$ a reference pressure. For instance, a $P_{\mathrm{e}}$ of $200 \mathrm{~Pa}$ has an SPL of $200 \mathrm{~dB}$ re $1 \mu \mathrm{Pa}$, whereas a $P_{\mathrm{e}}$ of $1 \mathrm{~Pa}$ has an SPL of $120 \mathrm{~dB}$ re $1 \mu \mathrm{Pa}$.

\section{Wave sounds recorded under the sand on a beach}

Sounds of waves approaching the shore were recorded on the beach during several summer days. A hydrophone was buried amidst the clam population at the same depth $(2-7 \mathrm{~mm})$ to which these clams burrow.

The hydrophone was attached to a tape recorder that had two input-channels: one channel recorded wave sounds; the other channel simultaneously recorded an observer's verbal description of the waves. Wave sounds were thus matched with particular events in the waves. Wave sounds were plotted as pressure versus time or as frequency spectra calculated from 0.5 -s intervals starting between 0.5 and $1 \mathrm{~s}$ before the arrival of swash at the hydrophone.

\section{Tidal variation in responsiveness of clams to sounds}

Clams were collected from the beach, transported to the laboratory, and placed in the aquarium. There they were exposed to natural light and dark cycles through a large window on one side of the room, but the fluorescent room lights were kept on constantly day and night to allow observation of clam behavior. About once each hour for several days, sounds were produced by knuckle knocking that was continued until no new clams emerged for $30 \mathrm{~s}$. The number of clams emerging in response to each knocking stimulus was counted. A hydrophone planted in the sand in the middle of the aquarium recorded the sounds for subsequent spectral analysis. This protocol was repeated for several collections of between 100 and 170 clams each.

Collections were made on two types of days. During the first type, high tide was between noon and midnight and between midnight and noon; during the second type, high tide coincided with midnight and noon. Thus the two types of days have noon and midnight falling either in or out of phase with high tide. Comparison of temporal patterns of responsiveness on the two types of days indicates whether there is a tidal or a daily rhythm of responsiveness.

A second experiment was performed according to the same protocol except that the window was blocked off, excluding sunlight and moonlight, and the room lights were turned off except for a few seconds during sampling periods.

\section{Clams' responsiveness to sounds from waves}

Clams were collected from the beach and placed in the aquarium in front of the speaker, where they dug into the sand. Recorded sounds of waves approaching the beach (about 200 successive swash) were presented to these clams during a 45-min period starting around the time of high tide. Clam responses were observed. A hydrophone in the sand $5 \mathrm{~cm}$ in front of the speaker monitored the loudness of the stimuli reaching the clams. This protocol was repeated on three summer days in an experiment involving a total of 210 clams.

\section{Frequency and amplitude specificity of responses}

To test specificity of responses to sounds, I subjected clams buried in the sand in the aquarium to specific synthesized sound stimuli of different frequencies and loudnesses. Sounds were presented using the underwater loudspeaker. Sounds were (i) broadband low-frequency sound, (ii) a pure low-frequency tone (with quieter, higher frequency harmonics), and (iii) a pure high-frequency tone (with quieter, higher frequency harmonics). Each presented sound was recorded by a hydrophone buried in the sand $5 \mathrm{~cm}$ in front of the speaker. The RMS sound pressures $\left(P_{e}\right)$ and frequency spectra were calculated.

Eighty clams were collected on the beach $30 \mathrm{~min}$ before high tide, transported to the laboratory, and placed hap- 


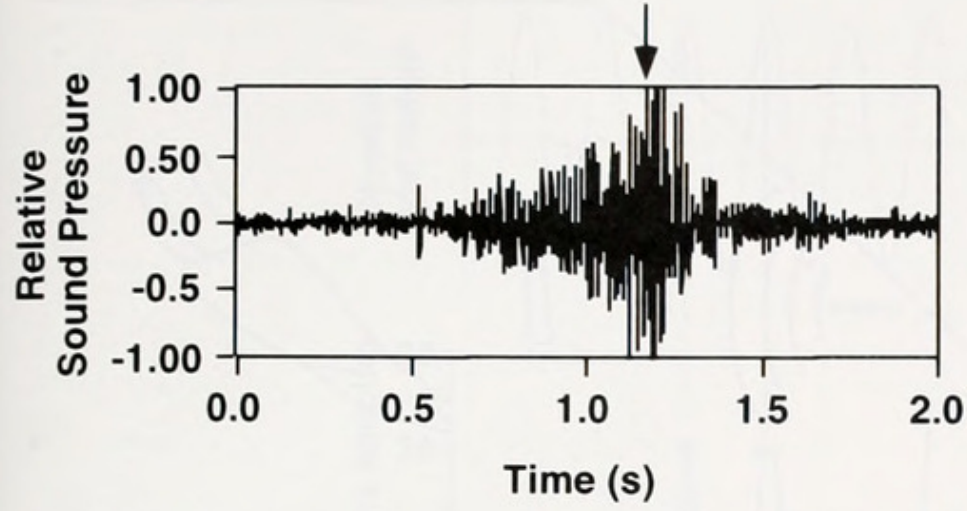

Figure 1. The sound of a wave approaching a beach as recorded from a hydrophone buried in the sand amidst the clams. Sound is represented as pressure versus time, with larger excursions of the pressure trace representing louder sounds. Relative sound pressure is relative to the highest pressure occurring in this trace. During the time preceding a wave's arrival at the hydrophone, a low-frequency rumble of increasing loudness is heard, culminating in a sudden increase in loudness when the wave reaches the hydrophone (arrow). Between waves there is relative silence.

hazardly in the aquarium, between the front of the speaker and the far end of the aquarium. Sounds were played to the clams during a $35-\mathrm{min}$ period starting $10 \mathrm{~min}$ after high tide.

The sounds were presented to the clams at regular intervals ( 1 min silence between presentations); the signal was always of $22 \pm 1$ s duration. Each sound was played at a variety of intensities seven times in a row. The highfrequency signal was played first, then the broadband lowfrequency signal, then the pure tone low-frequency signal. The number of clams jumping out of the sand for each stimulus was recorded. Significances of differences in responses were analyzed by regression analysis with dummy variables (Weisberg, 1980). (Statistical significance, throughout this paper, is considered to occur when the probability that the null hypothesis is true is less than 0.05.)

On two other days, additional experiments were performed according to a protocol that was identical except for the following. Instead of the three stimuli described above, a variety of synthesized low-frequency stimuli in the range of $20-100 \mathrm{~Hz}$ were presented. The stimuli were presented at irregular, more widely spaced intervals in random order and over a longer period of time (starting $40 \mathrm{~min}$ after and ending $3 \mathrm{~h}$ and $15 \mathrm{~min}$ after high tide). The number of clams responding was analyzed as a function of loudness, $\log \left(P_{e}\right)$, and time after high tide.

\section{Attenuation of sound and frequency responsiveness}

The results of the experiments on frequency and amplitude specificity must be interpreted relative to the frequency-specific attenuation of sound. Attenuation in the aquarium was measured by placing a hydrophone in the sand at $4,10,20,30$, and $40 \mathrm{~cm}$ in front of the speaker. Four pure tones, covering the range of frequencies used as stimuli, were individually played by the speaker. Attenuation of sound was calculated for each as the ratio of $P_{e}$ at each distance divided by $P_{e}$ at $4 \mathrm{~cm}$ from the speaker.

\section{Results}

\section{Wave sounds recorded under the sand on a beach}

Recorded wave sounds had characteristic patterns of loudness and frequency. A bore approaching the shore made a low rumbling sound that became louder as the bore approached the hydrophone (Fig. 1). Bigger bores made louder sounds than smaller ones as they approached the hydrophone. The rumbling noise of an approaching bore consists predominantly of low frequencies ranging from 40 to $300 \mathrm{~Hz}$, with the largest amplitudes being in the range of 60 to $100 \mathrm{~Hz}$ (Fig. 2A).

When a bore or swash reached the hydrophone, there was an additional sudden increase in loudness (arrow in Fig. 1). After a bore reached the hydrophone, the frequency content shifted toward more high-frequency components. As a bore or swash continued beachward, the sound loudness diminished.

The backwash also produced a distinct sound. It was softer than the upwash, sounded gurgly and uneven in loudness, and had more high-frequency components than swash.

\section{Responses of clams to knocking sounds}

Knocking on the plywood table under the aquarium produced a low-frequency sound with frequencies similar

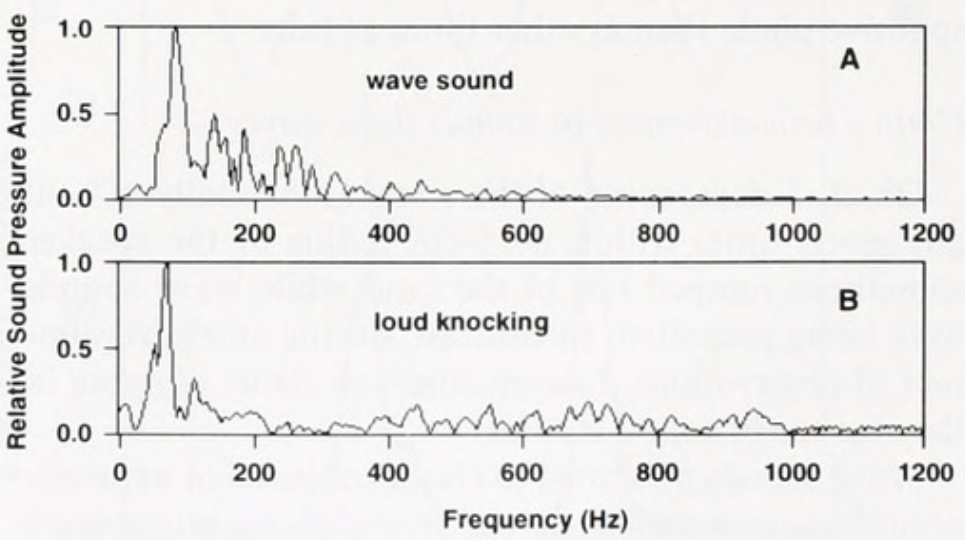

Figure 2. Comparison of frequency spectra of wave and knocking sounds that stimulate clams to jump. (A) A typical spectrum of a wave sound during a 0.5 -s interval that starts between 0.5 and $1 \mathrm{~s}$ before swash reaches the hydrophone. The rumbling sound that precedes the arrival of swash at the hydrophone consists mainly of low-frequency components. (B) The frequency spectrum of knocking sounds presented to clams. Knocking contains low-frequency sounds similar to those which precede the arrival of swash at a spot on the beach. 
to those of a bore approaching the beach (Fig. 2). Donax variabilis clams often responded to knocking by jumping out of the sand (Fig. 3). After a clam emerged from the sand, it fell on one valve or the other, often with siphon and foot extended. This behavior mimicked that of clams jumping out in front of swash on the beach. Clams lay on the sand for 3 to $30 \mathrm{~s}$ before digging in again. Occasionally, individual clams only partially emerged from the sand. Such hesitant clams could be induced to emerge completely by increasing either the loudness or the duration of knocking (suggesting that clams sum sound stimuli over time).

\section{Tidal variation in responsiveness of clams to knocking sounds}

The number of clams that jumped out of the sand depended on the time of tide, not on the time of day (Fig. 4a). Similar results were obtained when room lights were off and natural light cues were unavailable (Fig. 4b). The clams never jumped within 1 to $2 \mathrm{~h}$ of low tide; the number jumping increased as high tide approached, reached a maximum at high tide, and decreased after high tide. This tidal rhythm persisted in the absence of direct tidal cues (e.g., the aquarium's water level did not change), and independent of the light regime provided. The tidal rhythm persisted for three to five tidal cycles; subsequently no responses to sound could be elicited.

The maximum percentage of clams jumping at a given time was only $20 \%$. Jumping clams were not always the same individuals (individuals are recognizable by distinctive shell markings). Therefore, the $20 \%$ maximal response rate was not due merely to complete non-responsiveness in the other $80 \%$ of clams. Thus, individual clams become responsive and unresponsive several times per tidal cycle. At high tide, a larger fraction of the clams are in a responsive phase than at other times of tide.

\section{Clams' responsiveness to sounds from waves}

On all 3 days, some of the clams (maximally $20 \%$ at any given time) within a $15-\mathrm{cm}$ radius of the speaker sometimes jumped out of the sand while wave sounds were being presented. In contrast, during an entire summer of observations, I never observed clams jumping in the absence of sound stimuli.

Wave sounds presented to clams consisted of naturally occurring, quiet periods several seconds long, interspersed with the rumble of incoming waves. Whereas some wave sounds elicited responses, others not obviously different elicited no responses. Because of the complex nature of wave sounds, specific features to which clams responded could not be identified unambiguously. Nevertheless, jumps often coincided with the pre-arrival rumble. The loudness, $P_{e}$, of 0.5 -s samples of sound during pre-arrival

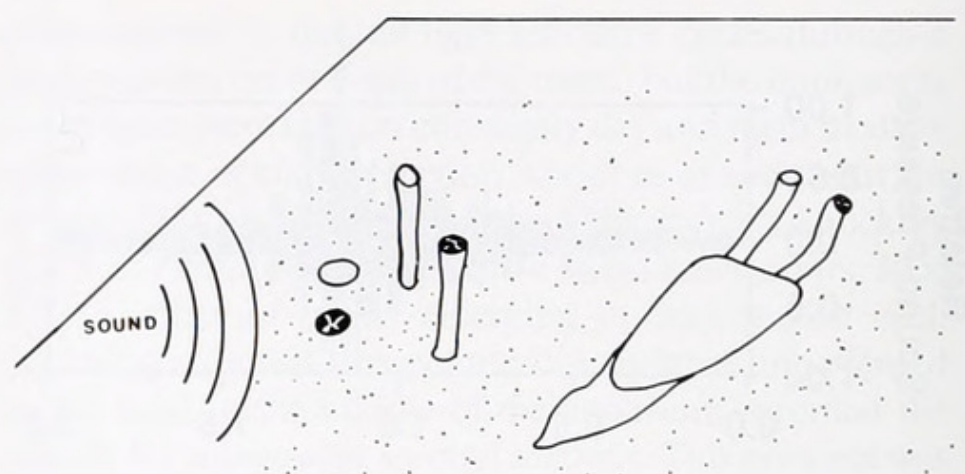

Figure 3. Observed responses of clams to sound stimuli. In a clam that did not visibly respond, only the clam's exhalant and inhalant (frilled) siphon were visible at the surface of the sand (at left). Clams responded either by elongating their siphons (middle), usually in response to a highfrequency $(832-\mathrm{Hz})$ tone, or they jumped out of the sand and lay with siphons and foot waving (at right). After 10-30 s, a clam that had jumped would again burrow into the sand.

rumbles was typically $30 \mathrm{~Pa}$, which is similar to the loudness of artificial sound stimuli that elicited the responses reported below.

\section{Frequency and amplitude specificity of responses}

Two separate behavioral responses to sound were observed-siphon elongation and jumping out of the sand (Fig. 3). Siphon elongation was qualitatively noted to occur primarily in response to higher frequencies (e.g., 500 and $832 \mathrm{~Hz}$ tones). Such elongation of the siphons was never seen in the absence of sound stimuli. This is the first report in the literature of siphon elongation as a response to sound, and its potential function is unknown.

The jumping responses were quantitatively analyzed. The frequency spectra of the sound stimuli are shown in Figure 5. At all frequencies, more clams jumped in response to louder sounds (Fig. 6). The number of clams jumping was linearly related to loudness as represented by $\log \left(P_{e}\right)$. For both the pure tone and the broadband low-frequency sounds, this linear relationship was tight (correlation $=0.98)$ and the slope was significantly nonzero $(P<0.001)$, whereas for the pure high-frequency tone, the relationship was less tight (correlation $=0.6$ ), and the slope was not significantly different from zero $(P$ $=0.1$ ). The frequency composition did not affect responsiveness; both pure tone and broadband low-frequency sounds elicited similar numbers of clams that jumped at a given loudness (low-frequency slopes not significantly different from each other). At a given loudness, more clams jumped in response to low- than to high-frequency sounds (slopes of the low-frequency sounds were significantly higher than the slope of the high-frequency tone). No clam was ever observed jumping in the absence of a sound stimulus. 

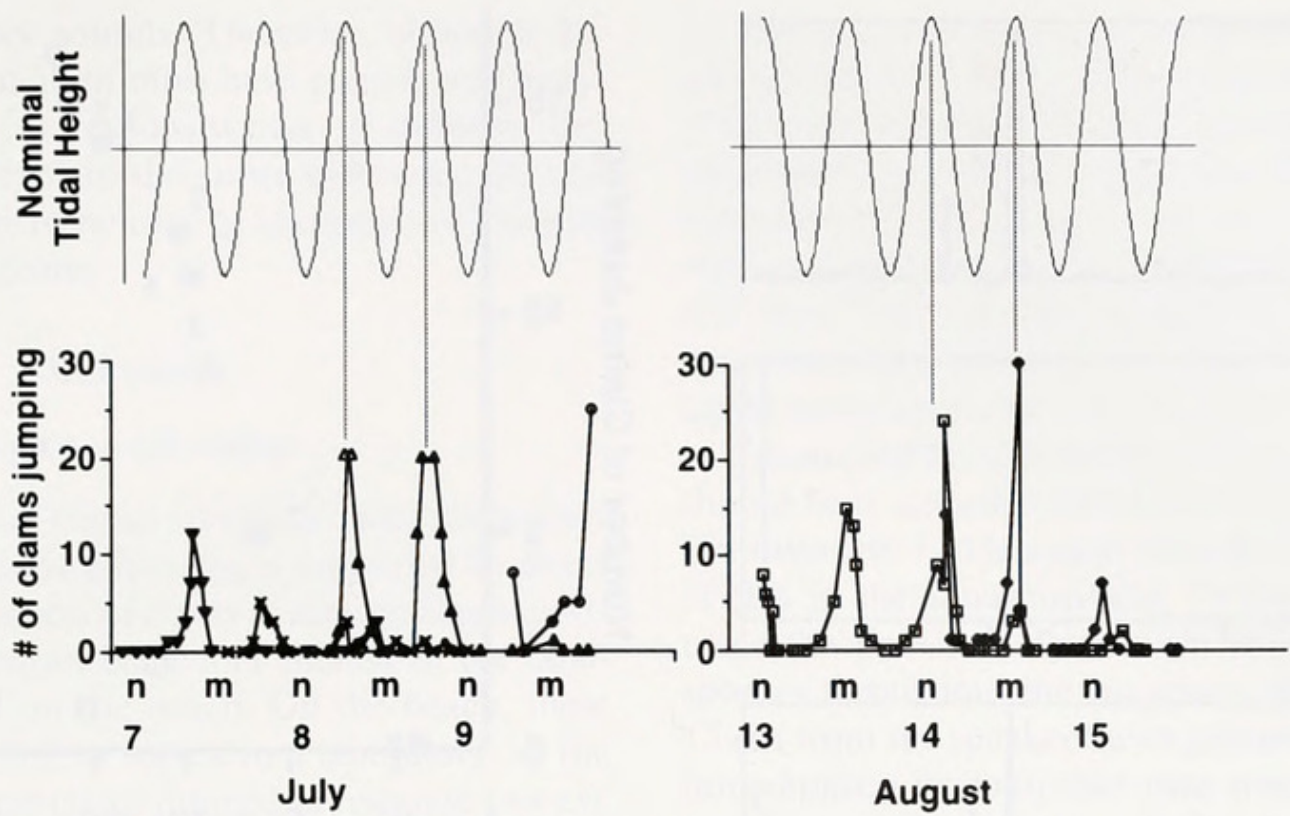

Figure 4a. Number of $D$. variabilis clams in the laboratory jumping in response to knocking sounds relative to time of day and tide. Noon is $\mathrm{n}$; midnight is $\mathrm{m}$. Clams were most responsive around the time of high tide and showed no response around the time of low tide. Clams responded for only three to five tidal cycles after they had been taken from the beach. The pattern shown is a compilation of several collections (represented by different symbols) taken on different days from the same beach. Room lights were continuously on in this experiment.

In the 2 additional days of experiments, performed during falling tides with several other synthesized lowfrequency stimuli, jumping responses were consistent with those already described. Specifically, the number of clams responding increased with loudness, $\log \left(P_{e}\right)$, and decreased with increasing time after high tide (multiple regression, $P<0.001$; loudness variable: $P<0.01$, time variable: $P<0.01, n=35$ ). A cumulative total of 147 responses were observed in 35 trials during those 2 days.

On these 3 days of experiments, clams responding to sounds jumped only if they were less than $14 \mathrm{~cm}$ from the speaker, and most responding clams were less than $5 \mathrm{~cm}$ from the speaker face. The proximity of responding clams to the sound source raises the possibility of nearfield effects (complicated wave interference patterns), which would complicate interpretation of the responses. A near field exists (close to a vibrating piston in an infinite baffle, an approximation to the geometry of the speaker: Kinsler et al., 1982, inter alia) only if the diameter $(0.2 \mathrm{~m})$ of the speaker is greater than the sound's wavelength. For an $832-\mathrm{Hz}$ sound the wavelength is $1.8 \mathrm{~m}$ (wavelength $=$ speed of sound divided by frequency; speed of sound in coarse silt sea bottoms assumed $=1540 \mathrm{~m} / \mathrm{s}$; Kinsler et al., 1982). The speaker is even smaller compared to the longer wavelengths of lower frequency sounds. Therefore, near-field effects are negligible in the present experiment. The spatial response pattern of clams must, however, also be considered with respect to frequency-dependent attenuation of sound.

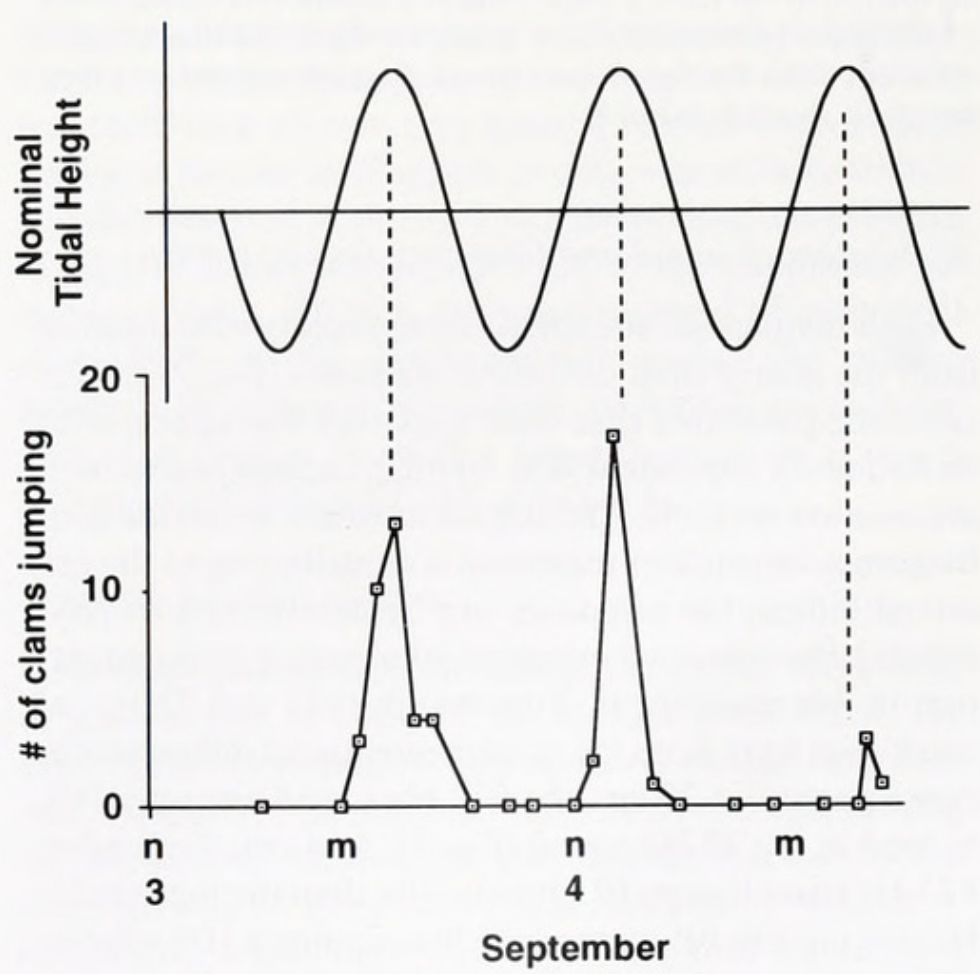

Figure 4b. Number of $D$. variabilis clams in the laboratory jumping in response to knocking sounds relative to time of day and tide. The results are similar to those shown in Figure $4 \mathrm{a}$, but in this experiment room lights were off except for a few seconds during sampling. 


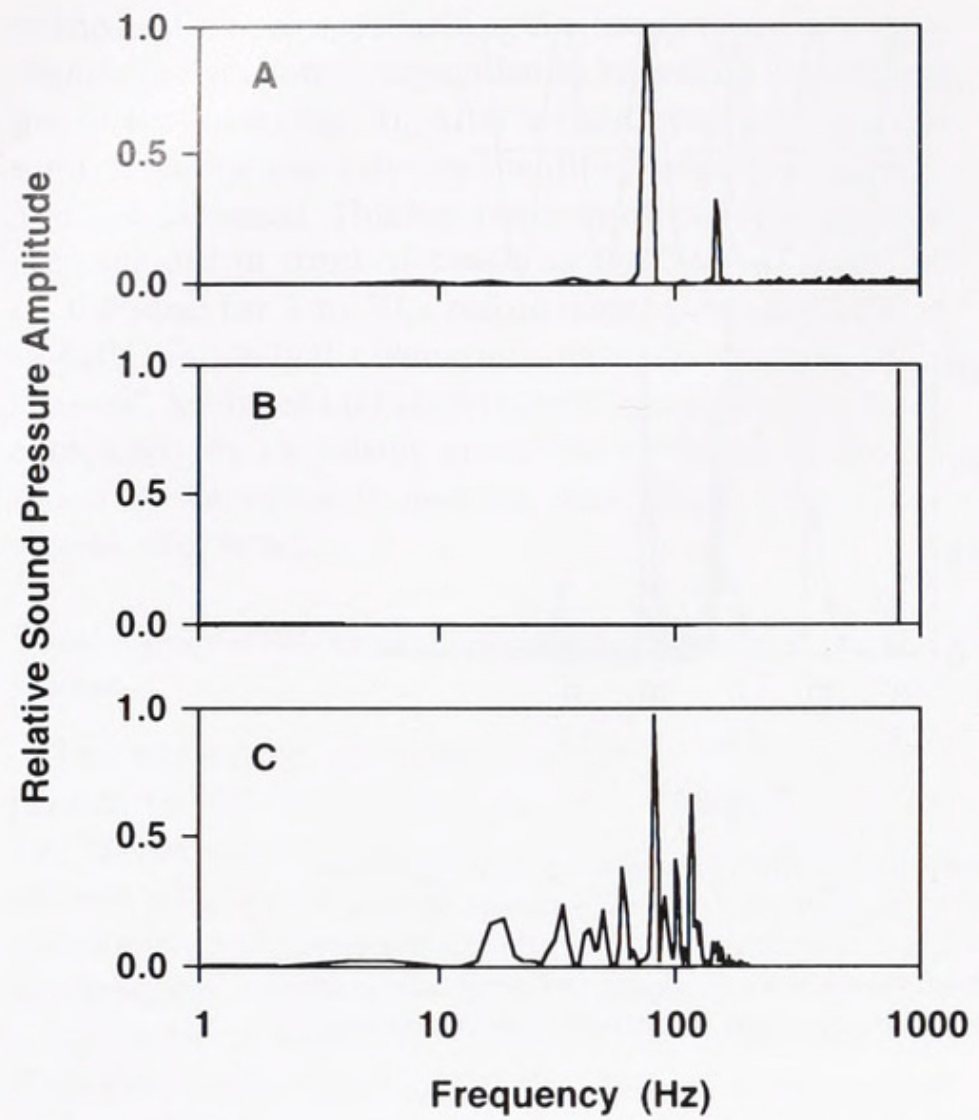

Figure 5. Frequency spectra of three artificially produced sounds presented to clams to test their responses to different sounds. The pure tone, low-frequency stimulus consisted of a $72-\mathrm{Hz}$ tone with a harmonic at $144 \mathrm{~Hz}(\mathrm{~A})$; the pure tone, high-frequency stimulus consisted of a 832 $\mathrm{Hz}$ tone (B) and a small harmonic at $1664 \mathrm{~Hz}$ (not shown); and the broadband, low-frequency noise had major frequency components ranging from 20 to $140 \mathrm{~Hz}(\mathrm{C})$. These frequency spectra were recorded with a hydrophone planted in the sand amidst the clams and thus represent stimuli to which the clams were exposed. Responses of clams to these sounds are shown in Figure 6.

\section{Attenuation of sound and frequency responsiveness}

High frequencies attenuated more rapidly with distance from the source than did low frequencies (Fig. 7), which raises the possibility that clam responses that appeared to be frequency dependent (Fig. 6) might actually have been attenuation artifacts. The degree to which attenuation of frequency-dependent responses is contributing to the observed differential responses can be determined by considering the measured extent of attenuation in the aquarium in this experiment. Two sounds $(832$ and $72 \mathrm{~Hz})$ of equal $P_{e}$ at $4 \mathrm{~cm}$ from the speaker attenuated differentially; consequently, at $30 \mathrm{~cm}$, the $832-\mathrm{Hz}$ sound was only $10 \%$ as loud as the $72-\mathrm{Hz}$ sound (Fig. 7). At $4 \mathrm{~cm}$, the loudest $832-\mathrm{Hz}$ stimuli were 10 times louder than the loudest 72 Hz stimuli (Fig. 6); conservatively assuming a $10 \%$ relative attenuation, at distances greater than $4 \mathrm{~cm}$, the loudest $832-\mathrm{Hz}$ sounds were at least as loud as the loudest $72-\mathrm{Hz}$ sounds. Yet only half to a third as many clams responded to the loudest high-frequency sounds as responded to the

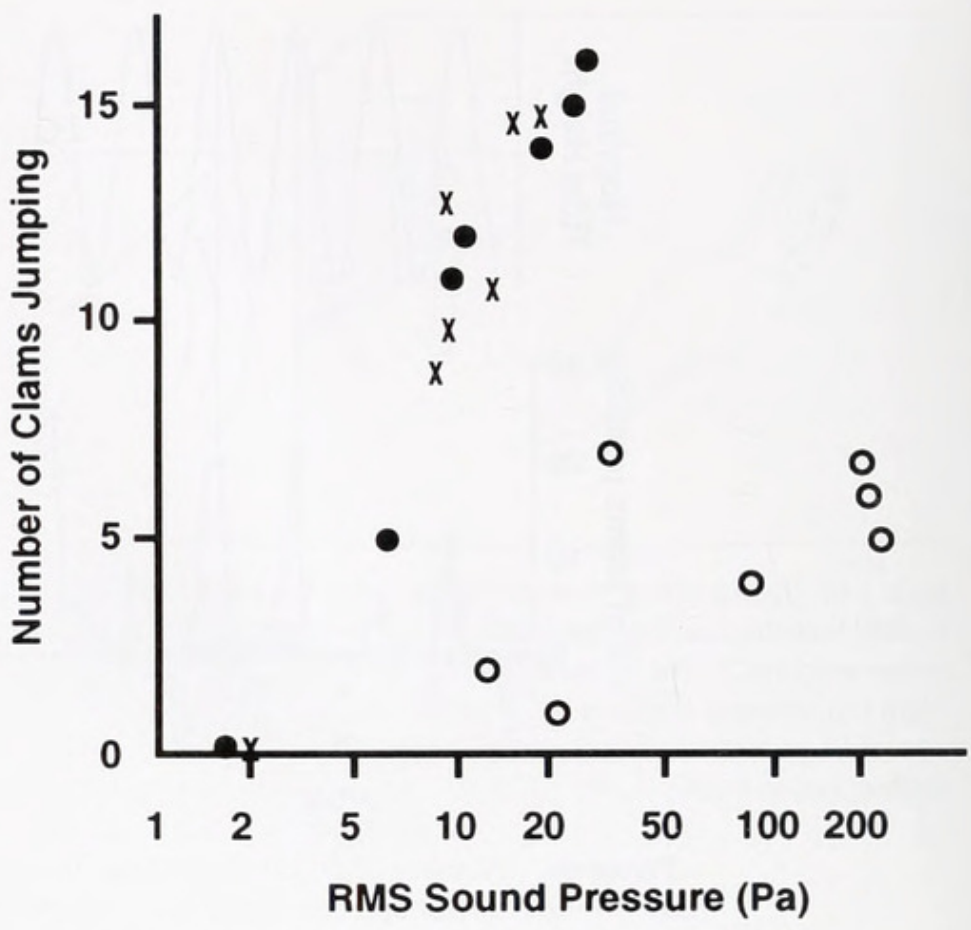

Figure 6. Number of clams jumping in response to sounds of different loudness and frequency. Louder sounds have higher root-mean-square (RMS) pressures. Three distinct frequency spectra (Fig. 5) were presented. More clams jump at a given loudness for both broadband $(20$ to $140 \mathrm{~Hz}$, $\times)$ and pure tone $(72-\mathrm{Hz}, \bullet)$ low-frequency sound than for a high-frequency $(832-\mathrm{Hz}, \mathrm{O})$ sound.

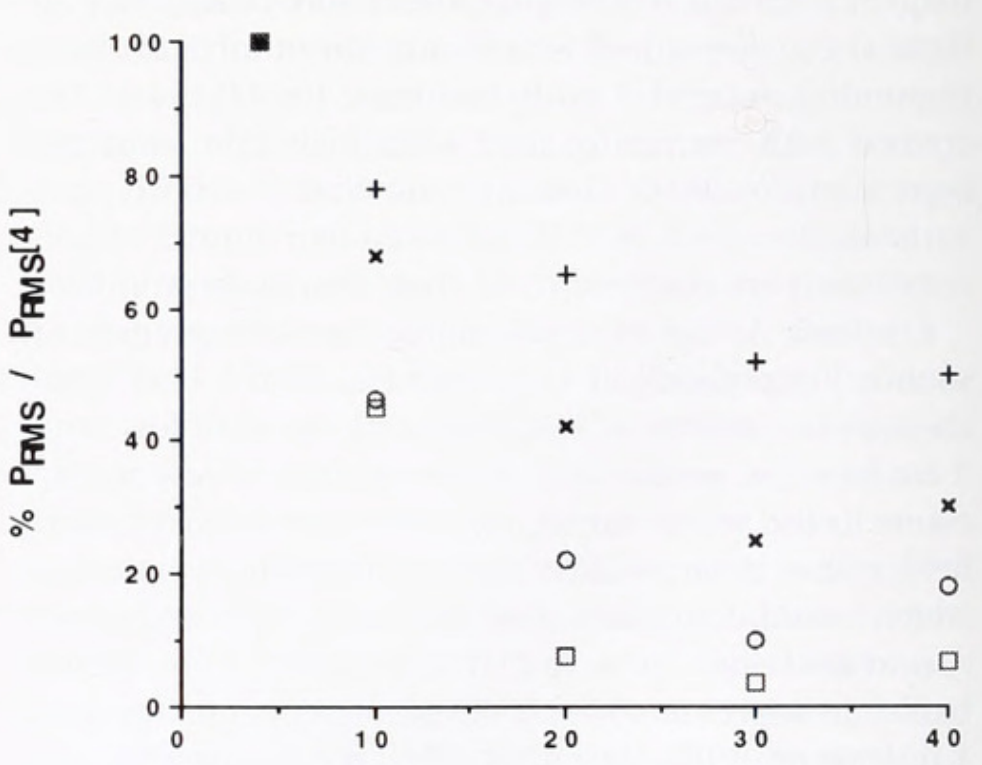

Distance From Speaker $(\mathrm{cm})$

Figure 7. Frequency-specific attenuation of sound in the sand in the aquarium in which acoustic tests were performed $(\square 832 \mathrm{~Hz} ; \bigcirc 500 \mathrm{~Hz}$; $\times 250 \mathrm{~Hz} ;+72 \mathrm{~Hz}$ ). The hydrophone was planted in the sand along the midline of the tank at several distances in front of the speaker. Attenuation is expressed as \% RMS sound pressure at the given distance from the loudspeaker divided by RMS sound pressure $4 \mathrm{~cm}$ from the face of the speaker. (The slight increase in sound at the point farthest from the speaker is probably due to sound patterns caused by partial reflection of sound waves off the aquarium wall.) 
loudest low-frequency sounds. Therefore, although differential sound attenuation must have contributed to the observed difference in responsiveness to different frequencies, it cannot explain the entire difference. $D$. variabilis clams are more responsive to low-frequency sounds in their jumping response.

\section{Discussion}

Wave sounds as cues for swash-riding

The proposition that Donax variabilis clams use sounds from waves as cues for swash-riding is supported by direct findings of responsiveness of clams to appropriate sounds and by parallels between behaviors elicited in the laboratory and observed on the beach. On the beach, these clams jump in response to waves; in a laboratory, in the absence of waves, these clams jumped in response to wave sounds and to artificially produced sounds that contained frequencies similar to those of wave sounds. Wave sounds recorded in the beach amidst coquina clams show distinct patterns of sound that allow the clams to predict the size and timing of incoming swash. For example, the loudness of a wave's sound predicts the excursion of that wave's swash. On the beach, clams preferentially jump for swash of large excursion (Ellers, 1995a); in the laboratory, clams were more responsive to louder sounds. Further correspondence between beach and laboratory is found in the observed endogenous tidal rhythm. Clams in the laboratory, which were isolated from direct tidal cues, were most responsive to sounds at high tide, and did not respond at all to sounds within 1 to $2 \mathrm{~h}$ of low tide. On the beach, clams did not jump out of the sand to ride waves within 1 to $2 \mathrm{~h}$ of low tide (Ellers, 1995a).

Taken together, these results indicate that sound is a major cue in determining whether these clams jump out of the sand to swash-ride. Loud sounds from large waves stimulate the clams to jump, and an endogenous rhythm of responsiveness modulates the jumping behavior and is thus a proximate cause of the characteristic tidal migration of these clams. This is the first report demonstrating use of flow-induced sounds by an invertebrate.

\section{Detection of pressure or velocity signals by clams}

An acoustic effect, associated with how close a responding clam is to the speaker, gives insight into possible mechanisms by which these clams may detect sounds. Sound can be detected as temporal changes in either pressure or velocity of the vibrating medium at a point. The hydrophone used in this experiment detects pressure. In contrast, organisms may use mechanisms (tiny hairs or cilia) that detect motion (velocity) rather than pressure. For a sound wave far from its source, pressure amplitude is directly proportional to velocity amplitude (Kinsler et al., 1982). For sound close to its source, however, velocity and pressure are not directly proportional, and velocity attenuates more rapidly than pressure. By convention, this effect is considered important (Bobber, 1970) if the distance from the source to the receiver (hydrophone or clam) is less than half a wavelength $(0.9$ and $11 \mathrm{~m}$ for $832-$ and $72-\mathrm{Hz}$ sounds, respectively). Therefore, in the area where the clams responded to sound, the velocity signal attenuated more rapidly than the pressure signal.

If clams had been detecting pressure, then louder sounds should have caused responses throughout the aquarium. For instance, $72-\mathrm{Hz}$ sound attenuated $P_{e}$ by a maximum of $50 \%$ in the aquarium (Fig. 7); therefore, a signal with twice the threshold $P_{e}$ at $4 \mathrm{~cm}$ should have caused responses throughout the aquarium. But clams more than $15 \mathrm{~cm}$ from the speaker never jumped; thus, they did not jump appropriately further away when the pressure signal was increased. Since the velocity signal attenuates more with distance than the pressure signal, the locations of responding clams suggest that the animals detected velocity rather than pressure. Other more complex possibilities exist, however, regarding the transmitting medium and the sensor used. For instance, scorpions have detectors both for compressional (sound) waves under the sand and for waves traveling on the interface between air and sand (Brownell, 1984).

\section{Population versus individual responses}

Loudness and frequency were not the only factors determining whether clams jumped in response to a sound. In fact, although clams jumped in connection with specific recorded wave sounds, they ignored many waves of equal loudness. Similar indifference to otherwise suitable stimuli was also observed in the rhythm experiments; a maximum of $20 \%$ of clams responded, even at the maximally responsive times. Perhaps the responsiveness of individual clams shifts several times per tidal cycle. The relative length or frequency of the responsive periods may change with time of tide, so that a larger fraction of clams is responsive at high tide than at low tide.

\section{Endogenous tidal rhythm}

An endogenous rhythm should persist for some time in the absence of cues. In the current experiment, the tidal rhythm in responsiveness persisted without tidal cues and irrespective of potential light cues, thus supporting the endogenous nature of the tidal rhythm. But the rhythm persisted only for three to five tidal cycles under laboratory conditions, suggesting either that the rhythm is a damped oscillator or that a clock is still running but its expression is blocked. For instance, lack of food may force cessation of jumping. Such small numbers of persisting cycles are sometimes observed for tidal rhythms; e.g., a tidal rhythm 
for phototaxis in larval grass shrimp persisted for only two to three cycles without cues (Douglass et al., 1992).

It was suggested that $D$. variabilis has no endogenous tidal rhythm (Tiffany, 1971) when clams transplanted to a beach with a different tidal cycle were observed following the new tidal cycle within $24 \mathrm{~h}$. Given the endogenous rhythm I observed (Fig. 4), an alternative interpretation is that the clock or damped oscillator was rapidly reset by the new conditions.

Additional stimuli on the beach may further modify responses in nature relative to those observed in the laboratory. For instance, the rhythm I observed was symmetrical during rising and falling tides. Clam responses were identical with respect to sound occurring any given time before and after high tide, at least within the limited ability of the present experiment to detect asymmetries. In contrast, observations on the beach show that some asymmetries in behavior exist. On the beach, clams jumped in advance of beachward-moving swash during the rising tide, but jumped into the backwash during the falling tide.

Asymmetries may be difficult to detect: behavior in the laboratory may not exactly mimic behavior on the beach because only partial cues are available. For instance, the relative amount of time that clams are covered by water may serve as a cue in nature. On a beach, the water level above the clams is constantly changing; in the aquarium, the water level remained unchanged.

Another natural cue that may be missing in the laboratory is the stimulus of being moved by a wave. In the aquarium, after a clam jumped out of the sand it lay on the sand and dug in again after 10-30 s. If immediately re-exposed to the same stimulus, a responsive clam often jumped out again (pers. obs.). Perhaps, on a beach, after a clam has been moved by a wave, its responsiveness to sound stimuli is reduced for some time.

\section{Usefulness of sound cues to marine animals}

Endogenous tidal rhythms are common in intertidal animals. Another swash-rider, the amphipod Synchelidium sp. (Enright, 1961a, 1963; Forward, 1980, 1986), has endogenous tidal rhythms in response to light (Forward, 1980) and pressure (Enright, 1961b). Endogenous tidal rhythms probably occur in other swash-riders: e.g., mole crabs, Emerita analoga (Cubit, 1969) and Remipes truncatifrons (Mori, 1938); and gastropods, Terebra salleana (Kornicker, 1961), Hastula inconstans (Miller, 1979), Bullia sp. (Ansell and Trevallion, 1969; McLachlan and Young, 1982), and Olivella biplicata (Johnson, 1966). Other Donax spp. that swash-ride (e.g., D. incarnatus and D. denticulatus: Ansell and Trueman, 1973; D. serra: Donn, 1987; and many others), may also respond to wave sounds on endogenous rhythms.
Whereas many populations of $D$. variabilis have been reported as migratory, at least during summer months, some populations either do not migrate or migrate only occasionally. There are many possible explanations for a cessation of migration, although none have been experimentally investigated. The role that sound plays in migration should be considered in that context. For example, one population of migratory $D$. variabilis that lived on steep-sloped beaches has been contrasted with another non-migratory population that lived on a more gently sloping beach (Mikkelsen, 1981). Perhaps waves on the gently sloping beach do not generate sounds that are sufficiently loud or sufficiently distinctive to produce selective jumping responses.

Finally, since sound and vibrational cues from waves are so obvious, and so obviously useful, even non-swashriding marine animals might use them. For instance, an urchin might tighten its grip on rocks in preparation for a particularly large wave, or an anemone might adjust its stiffness in preparation for a wave impact. I have observed both an anemone, Anthopleura elegantissima, and a sea urchin, Strongylocentrotus purpuratus, to respond to vibrations in the frequency range generated by waves.

In any case, the clams I studied are literally in tune with their environment.

\section{Acknowledgments}

This research is part of the author's Ph.D. dissertation completed at Duke University. I thank my thesis supervisor, S. Vogel, and committee members, V. L. Roth, E. J. Shaughnessy, V. A. Tucker, and S. A. Wainwright. NSERC postgraduate scholarships, Duke University teaching assistantships and a Cocos Foundation Training Grant in Morphology supported the author. Duke University Marine Laboratory provided facilities. I also thank S. A. Wainwright, who generously funded acoustic equipment; B. Hunnings, electronics technician, who built the sine-wave generator; and A. Johnson who critically read the manuscript.

\section{Literature Cited}

Ansell, A. D., and A. Trevallion. 1969. Behavioural adaptations of intertidal mollusks from a tropical sandy beach. J. Exp. Mar. Biol. Ecol. 4: 9-35

Ansell, A. D., and E. R. Trueman. 1973. The energy cost of migration of the bivalve Donax on tropical sandy beaches. Mar. Behav. Physiol. 2: $21-32$

Blaxter, J. H.S., and R.S. Batty. 1985. Herring behaviour in the dark: Responses to stationary and continuously vibrating obstacles. J. Mar. Biol. Assoc. U.K. 65: 1031-1049.

Bleckmann, H., T. Breithaupt, R. Blickhan, and J. Tautz. 1991. The time course and frequency content of hydrodynamic events caused by moving fish, frogs, and crustaceans. J. Comp. Physiol. A 168: 749757. 
Bobber, R. J. 1970. Underwater Electroacoustic Measurements. Naval Research Laboratory, Washington, DC.

Bradshaw, M. 1982. Bores and swash on natural beaches. Coastal Studies Unit Technical Report No. 82/4. Coastal Studies Unit, Department of Geography, The University of Sydney, Sydney, NSW, Australia.

Brownell, P. H. 1984. Prey detection by the sand scorpion. Sci. Am 251: 86-97.

Burrus, C. S., and T. W. Parks. 1985. DFT/FFT and Convolution Algorithms - Theory and Implementation. John Wiley and Sons, New York.

Cubit, J. 1969. Behaviour and physical forces causing migration and aggregation of the sand crab Emerita analoga (Stimpson). Ecology 50: $118-123$

Donn, T. E., Jr. 1987. Longshore distribution of Donax serra in two log-spiral bays in the eastern Cape, South Africa. Mar. Ecol. Prog. Ser. 35: 217-222.

Douglass, J. K., J. H. Wilson, and R. B. Forward. 1992. A tidal rhythm in phototaxis of larval grass shrimp (Palaemonetes pugio). Mar. Behav. Physiol. 19: 159-173

Ellers, O. 1987. Passive orientation of benthic animals in flow. Pp 45-68 in Signposts in the Sea, Proceedings of a Multidisciplinary Workshop on Marine Animal Orientation and Migration, W. F. Herrnkind and A. B. Thistle, eds. Dept. of Biological Science, Florida State University, Tallahassee.

Ellers, O. 1988. Locomotion via swash-riding in the clam Donax variabilis. Ph.D. Dissertation, Duke University, Durham, NC.

Ellers, O. 1995a. Behavioral control of swash-riding in the clam Donax variabilis. Biol. Bull. 189: 120-127.

Enright, J. T. 1961a. Distribution, population dynamics and behavior of a sand beach crustacean Synchelidium sp. Ph.D. Dissertation, University of California at Los Angeles.

Enright, J. T. 1961b. Pressure sensitivity of an amphipod. Science 133: $758-760$

Enright, J. T. 1963. Responses of an amphipod to pressure changes. Comp. Biochem. Physiol. 7: 131-145.

Forward, R. B. 1980. Phototaxis of a sand-beach amphipod: physiology and tidal rhythms. J. Comp. Physiol. 135: 243-250.

Forward, R. B. 1986. Behavioral responses of a sand-beach amphipod to light and pressure. J. Exp. Mar. Biol. Ecol. 102: 55-74.

Johnson, P. T. 1966. On Donax and other sandy beach inhabitants. Veliger 9: 29-30.

Karlsen, H. E. 1992a. The inner ear is responsible for detection of infrasound in the perch (Perca fluviatilis). J. Exp. Biol. 171: 163172.
Karlsen, H. E. 1992b. Infrasound sensitivity in the plaice (Pleuronectes platessa). J. Exp. Biol. 171: 173-187.

Kinsler, L. E., A. R. Frey, A. B. Coppens, and J. V. Sanders. 1982. Fundamentals of Acoustics, 3rd ed. John Wiley and Sons, New York.

Kornicker, L. S. 1961. Observations on the behaviour of the littoral gastropod Terebra salleana. Ecology 42: 207.

Kreithen, M. L., and D. B. Quine, 1979. Infrasound detection by the homing pigeon: A behavioral audiogram. J. Comp. Physiol. 129: 1-4.

Loesch, H. C. 1957. Studies on the ecology of two species of Donax on Mustang Island, Texas. Inst. Mar Sci. 4: 201-227.

McLachlan, A., and N. Young. 1982. Effects of low temperature on the burrowing rates of four sandy beach mollusks. J. Exp. Mar. Biol. Ecol. 65: 275-284

Mikkelsen, P. S. 1981. A comparison of two Florida populations of the coquina clam, Donax variabilis Say, 1822 (Bivalvia: Donacidae). I. Intertidal density, distribution and migration. Veliger 3: 230-239.

Miller, W. 1979. The biology of Hastula inconstans (Hinds, 1984) and a discussion of life history similarities among other Hastulas of similar proboscis type. Pac. Sci. 33: 289-306.

Mori, S. 1938. Characteristic tidal rhythmic migration of a mussel, Donax semigranosus Dunker, and the experimental analysis of its behaviour at the flood tide. Dobutsugaku Zasski, [=Zool. Mag. (Japan)] 50: $1-12$.

Mori, S. 1950. Characteristic tidal rhythmic migration of a mussel, Donax semigranosus Dunker, and the experimental analysis of its behaviour (II). Dobutsugaku Zasski [=Zool. Mag. (Japan)] 59: 8789.

Naylor, E. 1985. Tidally rhythmic behaviour of marine animals. Symp. the Soc. Exp. Biol. 39: 63-93.

Packard, A., H. E. Karlsen, and O. Sand. 1990. Low frequency hearing in cephalopods. J. Comp. Physiol. A 166: 501-505.

Tiffany, W. J. III. 1971. The tidal migration of Donax variabilis Say (Mollusca: Bivalvia). Veliger 14: 82-85.

Trueman, E. R. 1971. The control of burrowing and the migratory behaviour of Donax denticulatus (Bivalvia: Tellinacea). J. Zool., Lond. 165: 453-469.

Turner, H. J., Jr., and D. L. Belding. 1957. The tidal migrations of Donax variabilis Say. Limnol. Oceanogr. 2: 120-124.

Weisberg, S. 1980. Applied Linear Regression. John Wiley and Sons, New York.

Williams, B. G., J. D. Palmer, and D. N. Hutchinson. 1993. Comparative studies of tidal rhythms XIII. Is a clam clock similar to those of other intertidal animals? Mar. Behav. Physiol. 24: 1-14. 


\section{$2 \mathrm{BHL}$ Biodiversity Heritage Library}

Ellers, Olaf Walter Josef. 1995. "Discrimination Among Wave-Generated Sounds by a Swash-Riding Clam." The Biological bulletin 189, 128-137. https://doi.org/10.2307/1542463.

View This Item Online: https://www.biodiversitylibrary.org/item/17166

DOI: https://doi.org/10.2307/1542463

Permalink: https://www.biodiversitylibrary.org/partpdf/19919

\section{Holding Institution}

MBLWHOI Library

\section{Sponsored by}

MBLWHOI Library

\section{Copyright \& Reuse}

Copyright Status: In copyright. Digitized with the permission of the rights holder.

License: http://creativecommons.org/licenses/by-nc-sa/3.0/

Rights: https://biodiversitylibrary.org/permissions

This document was created from content at the Biodiversity Heritage Library, the world's largest open access digital library for biodiversity literature and archives. Visit BHL at https://www.biodiversitylibrary.org. 